\title{
Diabetes Autoantibodies Mediate Neural- and Endothelial Cell- Inhibitory Effects Via 5-Hydroxytryptamine- 2 Receptor Coupled to Phospholipase C/Inositol Triphosphate/Ca2+ Pathway
}

\author{
Mark B. Zimering * \\ Endocrinology, Veterans Affairs New Jersey Healthcare System, East Orange, New Jersey; Rutgers-Robert Wood Johnson Medical School, New \\ Brunswick, New Jersey.
}

Received: September 05, 2017; Accepted: September 19, 2017; Published: October 04, 2017

*Corresponding author: Dr. Mark B. Zimering, Endocrinology, Veterans Affairs New Jersey Healthcare System, East Orange, New Jersey; RutgersRobert Wood Johnson Medical School, New Brunswick, New Jersey; Fax: 908 604-5249;E-mail: mark.zimering@va.gov

\begin{abstract}
Aims: To identify the G-protein coupled receptor(s) on neuroblastoma and endothelial cells which mediate neural- and endothelial cellinhibitory effects in plasma autoantibodies from a subset of older type 2 diabetes with neurologic and vascular co-morbidity. To determine the mechanism(s) of neurite retraction induced by diabetic pathologies' auto antibodies.

Methods: Protein-A eluates from plasma of 11 diabetic patients having nephropathy, moderate-severe obesity and/or complications in which increased inflammation plays a role (depression, Parkinson's disease, atrial fibrillation, obstructive sleep apnea) were tested for neurite retraction and decreased survival in N2A neuroblastoma cells, and decreased survival in pulmonary artery endothelial cells. Specific antagonists of G protein coupled receptors belonging to the $\mathrm{G}$ alpha $\mathrm{q}$ subfamily of hetero trimetric $\mathrm{G}$ proteins or the phospholipase $\mathrm{C} / \mathrm{inositol}$ triphosphate/Ca2+ pathway were tested for modulatory effects on diabetic pathologies' autoantibody-induced N2A neurite retraction, or cell survival.

Results: Co-incubation with specific antagonists of the 5-hydroxytryptamine- $2 \mathrm{~A}$ receptor significantly prevented acute N2A neurite retraction induced by 50-100 nM concentrations of diabetic pathologies' autoantibodies. Protection against neurite retraction (M100907> spiperone> ketanserin) closely paralleled the antagonists' potency order at the 5-HT2-AR. Neuroblastoma or endothelial cell death (after 24 hours incubation)

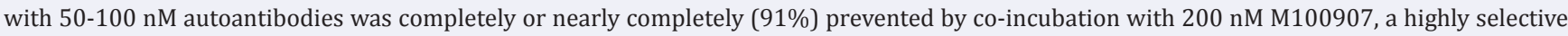
5-HT2-AR antagonist. Alpha-1 adrenergic, angiotensin II, metabotropic glutamate 5, or endothelin A (100 nM-10 $\mu$ M) receptor antagonists did not substantially inhibit autoantibody-induced cell death. The intracellular calcium chelator (BAPTA-AM, $50 \mu \mathrm{M}$ ) and inhibitors of the inositol triphosphate (IP3) receptor (2-APB, $50 \mu \mathrm{M})$, and phospholipase C-gamma $(\mathrm{U} 73144,1 \mu \mathrm{M})$ each significantly protected against autoantibody-induced acute N2A neurite retraction.
\end{abstract}

Conclusion: These data suggest that neural- and endothelial- inhibitory effects in autoantibodies from older adult diabetes with nephropathy and obesity/inflammation-associated complications are mediated by agonist autoantibodies directed against the 5-hydroxytryptamine 2 receptor positively coupled to the phospholipase C/inositol triphosphate/ cytosolic Ca2+ release pathway.

Keywords: 5-hydroxytryptamine-2 receptor; diabetes; autoantibodies; neurovascular diseases

\section{Introduction}

Obesity and inflammation are risk factors for certain diabetes-related neurovascular complications although the underlying mechanism is unclear. Diabetic nephropathy is characterized by increased micro vascular permeability [1] (e.g. micro- and macro-albuminuria) and inflammation thought to drive the progression to end-stage-renal disease (ESRD) [2]. In a prior study in 305 diabetic nephropathy patients, we reported that albuminuria interacted significantly with endothelial cell inhibitory autoantibodies in predicting the risk of substantial decline in renal function, ESRD or death during three-five years' randomized treatment in the VA NEPHRON D- a clinical trial comparing angiotensin receptor blocker (ARB) alone vs. combined $\mathrm{ARB}$ and angiotensin-converting enzyme inhibitor medications $[3,4]$. Endothelial cell (EC) inhibitory autoantibodies were also increased in subsets of diabetic patients suffering with specific co-morbidities including: diabetes, schizophrenia and the comorbid clusters: painful neuropathy/maculopathy/nephropathy obstructive sleep apnea/atrial fibrillation/dementia and primary open-angle glaucoma/dementia [5-9]. Mechanistically, potent endothelial cell autoantibodies caused apoptosis through a mechanism involving large, sustained increases in intracellular $\mathrm{Ca} 2+$ leading to caspase-3 activation [10]. Diabetic depression 
autoantibodies caused neurite retraction, and prolonged N2A neuroblastoma cell depolarization [8]. A subset of dementia/ glaucoma autoantibodies caused contraction and process retraction in cerebral cortical astrocytes [9]. The pleiotropic actions of the diabetic neurovascular autoantibodies led us to test (in the present study) whether endothelial cell- and neuro toxic diabetic autoantibodies may target a specific subclass of G-protein coupled receptors (GPCR) linked to increases in intracellular $\mathrm{Ca} 2+$.

\section{Participants \& Methods}

\section{Participants}

Informed consent for the Investigational Review Boardapproved, local, Veterans Affairs New Jersey Healthcare System proposal was obtained from all study patients prior to blood drawing. Baseline clinical characteristics in the study participants are shown in (Table 2). The cohort was comprised of older men, having a mean age of 68 years, average BMI of $38 \mathrm{~kg} / \mathrm{m} 2$, average duration of type 2 diabetes of 13.8 years, and mean glycosylated hemoglobin of $7.6 \%$. In order to explore possible linkages between autoantibodies, obesity, inflammation and diabetic neurodegenerative or vascular complications, patients were selected on the basis of a high concordance (in individual patients) of these same risk factors and complications. Overall, the cohort had a high prevalence of neurodegenerative disorder(s) (64\%), [i.e. major depression ( $n=5)$, Parkinson's disease $(n=2)$, glaucoma $(\mathrm{n}=1)]$, nephropathy $(82 \%)$, moderate-or severe-risk obesity (82\%), and one or more conditions linked to inflammation, e.g. atrial fibrillation (64\%), autoimmune thyroid disease (27\%), obstructive sleep apnea (45\%). Nine of eleven moderate-severely obese patients had an average of two or more complications (atrial fibrillation, obstructive sleep apnea, painful neuropathy) associated with inflammation. Our underlying hypothesis is that diabetic nephropathy drives autoantibody production through the release of endothelial cell auto antigens in the setting of chronic inflammation. As a test of this hypothesis, all but two study patients had diabetic nephropathy. Moderate-severely obese patients were selected for this mechanistic study, since in prior reports, obesity was significantly associated with pathologies (e.g. atrial fibrillation, obstructive sleep apnea) in which neurotoxic plasma autoantibodies were present at increased levels compared to control diabetic patients [8].

Table 1: Autoantibodies in diabetes having co-morbid neurovascular complications: spectrum of autoantibody- mediated effects [5,6,7,8,9]

\begin{tabular}{|c|c|c|c|}
\hline Complication & Autoantibodies & Autoantibodies Endothelial cell & Neurons \\
\hline Nephropathy & Incr vs. control & Inhibit/stim survival & Neurite retraction, depolarization \\
\hline Painful Neuropathy & Incr vs. control & Inhibit survival & Neurite retraction \\
\hline Depression & Incr vs. control & Inhibit survival & Neurite retraction, astrocytes effects* \\
\hline Glaucoma & Incr vs. control & Inhibit survival & Neurite rtrct/outgrowth [30] \\
\hline Parkinson' disease & Incr vs. control & Inhibit/stim survival & NT neurite retraction, depolarization \\
\hline Obstr sleep apnea & Inc vs. control & NT & N2A depolarization \\
\hline Atrial fibrillation & Co-morbid with OSA & Inhibit & N2A neurite retraction, depolarization \\
\hline Schizophrenia & Incr vs. control & N & N \\
\hline
\end{tabular}

*Autoantibodies in diabetic primary open-angle glaucoma and dementia caused contraction and process shortening in cerebral cortical astrocytes [9]. Obstr (uctive); Incr (eased) compared to Control diabetes populations lacking this complication; stim (ulate); NT- not tested; N2A mouse Neuroblastoma cell line; rtrct (retraction), cntr (contraction); short (ening), depol (arization), DG dentate gyrus, NPC-neural progenitor cell, "i.e. "NPC-neural progenitor cell, OSA- obstructive sleep apnea.

\section{Diagnostic methods and subgroups}

\section{Depression}

All patients having a diagnosis of major depression were evaluated by psychiatry staff at the Veterans Affairs New Jersey Health Care System as previously reported [5]. Painful diabetic neuropathy is defined according to previously reported criteria [7]. Diabetic nephropathy is defined as persistent micro-or macro-albuminuria, the latter urinary albumin excretion > $300 \mathrm{mg} / \mathrm{g}$ creatinine or urinary protein excretion $>500 \mathrm{mg} / \mathrm{g}$ creatinine.

\section{Morbid obesity}

Morbid obesity is defined as body mass index (BMI) $>40 \mathrm{~kg} /$ $\mathrm{m}^{2}$. Moderate-severe obesity is defined as BMI $>35 \mathrm{~kg} / \mathrm{m}^{2}$. Pt 1: A 71 -year- old male with BMI $48 \mathrm{~kg} / \mathrm{m}^{2}$, diabetic nephropathy, atrial fibrillation, obstructive sleep apnea (OSA), glaucoma, depression, and painful neuropathy.

\section{Parkinson's disease (PD)}

PD 1: A 70- year- old male with diabetic nephropathy, atrial fibrillation, obstructive sleep apnea, Parkinson's disease and painful neuropathy.

PD 2: A 79 -year- old male with diabetic nephropathy, autoimmune thyroid disease, and Parkinson's disease.

\section{Cell Culture}

Bovine pulmonary artery (BPA) endothelial cells (Sigma, St. Louis, MO) were maintained at $37 \mathrm{C}$ in 5\% CO2/95\% air in Medium 199 plus 10\% fetal calf serum. BPA endothelial cells were passaged continuously and used between passages 4-10.

\section{Endothelial Cell Growth Assays}

Endothelial cell number assays were carried out as previously reported [11]. After 48 hours' incubation in the presence of protein-A-eluate fractions, cell number, i.e. cell-associated acid phosphatase activity, was determined as previously described 
[11]. Growth-promoting activity (determined in quadruplicate for each test factor) is expressed as a percentage of the control cell number (determined in quadruplicate) for cells grown in the absence of protein-A- eluate fractions.

\section{Protein A Affinity Chromatography}

Protein A chromatography was carried out as previously described [11]. Eluate fractions were stored at 0-4 degrees C. All fractions were sterile filtered (Millipore Corp., Bedford, MA; $0.22 \mathrm{um}$ ) before assay for growth-promoting activity.

\section{Mouse N2A cells}

Mouse N2A neuroblastoma cells were cultured as previously described [6].

\section{Neurite retraction assays}

Mouse N2A cells were plated at low density in $35 \mathrm{~mm}$ dishes. After one to two days incubation at 37 degrees C, protein A-eluate fractions $(7-10 \mu \mathrm{g} / \mathrm{mL})$ from diabetic pathologies' plasma were added in the presence or absence of GPCR antagonists or antagonists of downstream signaling molecules or an intracellular calcium chelator. Thin neurite length was estimated (at baseline and again after 25 minutes exposure to added fractions) as the average length in all thin neurites (having diameter of $\sim 0.5-1$ microns) extending at least 2 cell diameters from the cell body at baseline in each of 5-10 cells. Percent of antibody-induced neurite retraction is expressed as a percent of acute neurite lengthshortening (observed after 25 minutes) in cells exposed to test factors plus autoantibodies compared to identical concentration of same autoantibodies alone. Each point represents the mean of two or more determinations which generally varied by $<15 \%$.

\section{N2A survival assays}

N2a cells were plated at $10^{3}-10^{4}$ cells $/ \mathrm{cm}^{2}$ in 96 cell plates in DMEM plus $10 \%$ fetal calf serum. After 2-3 days incubation, test

Table 2: Baseline clinical characteristics in study cohort

\begin{tabular}{|c|c|}
\hline & Risk factor \\
\hline Age (years) & $67.8 \pm 7.0$ \\
\hline Body mass index $\left(\mathrm{kg} / \mathrm{m}^{2}\right)$ & $38.2 \pm 5.8$ \\
\hline Diabetes Duration (years) & $13.8 \pm 5.9$ \\
\hline HbA1c (\%) & $7.6 \pm 1.4$ \\
\hline Nephropathy (yes/no) & $(9 / 2)$ \\
\hline Atrial fibrillation (yes/no) & $(7 / 4)$ \\
\hline Obstructive sleep apnea (yes/no) & $(5 / 6)$ \\
\hline Painful neuropathy (yes/no) & $(7 / 4)$ \\
\hline Autoimmune thyroid disease (yes/no) & $(3 / 8)$ \\
\hline Neurodegenerative disease* (yes/no) & $(7 / 4)$ \\
\hline * Depression (n=5), Parkinson's disease (n=2). \\
\hline
\end{tabular}

fractions were added to cells in quadruplicate. Following (8 or 24 hours) incubation at 37 degrees $\mathrm{C}$, cell survival was determined using MTT assay. Twenty micro liters of a $5 \mathrm{mg} / \mathrm{mL}$ MTT solution in PBS was added to each well; after 3.5 hours incubation at 37 degrees C, MTT solution was discarded and 200 micro liters DMSO was added to each well. After 10 minutes, absorbance was determined at $570 \mathrm{~nm}$. Cell survival is expressed as a percentage of the absorbance in control N2A cells to which no test factors were added.

\section{Adult neural progenitor cells}

The stem cells were originally isolated from the dentate gyrus of adult Fisher 344 rats and were obtained from Dr. Fred Gage at the Salk Institute. The NPCs were cultured as previously described [5]. Test protein-A eluate fractions from diabetic pathology plasma (5-7 $\mu \mathrm{g} / \mathrm{mL}$ protein) was added to cells on DIV 3 . Acute process shortening and cell contraction were determined by continuous observation (at 5 minute intervals) using a Nikon inverted microscope at 200X magnification for up to 75 minutes after adding test factors.

\section{Chemicals}

M100907, ketanserin, spiperone, DOI, bosentan, losartan, prazosin, U73211, BAPTA-AM, 2-APB were obtained from Sigma Chemical Co. (St Louis, MO.) All other chemicals and reagents were analytical grade.

\section{Protein Determinations}

Protein concentrations were determined by a bicinchoninic acid protein assay kit (Pierce Chemical Co., Rockford, IL).

\section{Statistics}

All data are the mean \pm SD (Tables 1, 2, 4) or SEM (Figure 4-7) as indicated. Comparisons were made by Student's t-test for a continuous variable with a significance level, $p=0.05$.

Table 3: Pharmacologic profile of endothelial cell-inhibitory activity in diabetes autoantibodies

\begin{tabular}{|c|c|c|}
\hline Antagonist [Conc] & GPCR & $\begin{array}{c}\text { \% Neutralization of Diab } \\
\text { Autoantibody-induced EC cell } \\
\text { death }\end{array}$ \\
\hline M100907 $[2 \mu \mathrm{M}]$ & 5-HT2a/b/c & $100 \%$ \\
\hline M100907 [200 nM] & 5-HT2a/b/c & $80 \%$ \\
\hline M100907 [100 nM] & 5-HT2a/b/c & $22 \%$ \\
\hline Ketanserin $[100 \mathrm{nM}]$ & 5-HT2a/c & $0 \%$ \\
\hline Prazosin $[1 \mu \mathrm{M}]$ & A1-AR & $0 \%$ \\
\hline Losartan $[10 \mu \mathrm{M}]$ & A1-1R & $23 \%$ \\
\hline Bosentan $[10 \mu \mathrm{M}]$ & ET-AR & $17 \%$ \\
\hline CTEP $[10 \mu \mathrm{M}]$ & mGlu-5R & $15 \%$ \\
\hline
\end{tabular}

A1-AR- alpha 1 adrenergic receptor; AT-1R- angiotensin II, type 1 receptor; ET-AR- endothelin A receptor, mGlu-5R- metabotropic glutamate 5 receptor 


\section{Ca2+ Pathway}

Table 4: Effect of antagonists of the PLC/IP3R/Ca2+ pathway on autoantibody- induced N2ANeurite retraction

\begin{tabular}{|c|c|c|}
\hline Treatment & [Conc] & $\begin{array}{c}\text { \% Diab Autoantibody-induced } \\
\text { neurite retraction* }\end{array}$ \\
\hline Diabetic autoantibody & $50 \mathrm{nM}$ & $100 \pm 0 \%$ \\
\hline Y27632 & $10 \mu \mathrm{M}$ & $-12 \pm 3 \%$ \\
\hline U73122 (PLC inhibitor) & $1 \mu \mathrm{M}$ & $12 \pm 4 \%$ \\
\hline $\begin{array}{c}\text { BAPTA-AM (Ca2+ } \\
\text { chelator) }\end{array}$ & $30 \mu \mathrm{M}$ & $0 \pm 0 \%$ \\
\hline 2-APB (IP3R inhibitor) & $50 \mu \mathrm{M}$ & $-14 \pm 7 \%$ \\
\hline
\end{tabular}

*Neurite retraction was determined as percent of acute neurite shortening (after 25 mins Exposure) in the presence of $50 \mathrm{nM}$ diabetic autoantibodies alone, or following a brief pre incubation (10 mins) with indicated concentrations of specific antagonists of the PLC/IP3/Ca2+ path way or RhoA/ROCK signaling pathway. Results are mean \pm SD in two or more experiments.

\section{Results}

Effect of diabetic autoantibodies in rat dentate gyrus neural progenitor cells Rat dentate gyrus (DG) neural progenitor cells (NPC) were sensitive to low concentrations of diabetic depression autoantibodies (5-7 $\mu \mathrm{g} / \mathrm{mL})$ and were previously reported to cause both NPC process retraction and decreased cell survival [5]. In the present study, diabetic autoantibodies $(8 \mu \mathrm{g} / \mathrm{mL}=80$ $\mathrm{nM}$ ) from a patient suffering with nephropathy, Parkinson's disease, atrial fibrillation and painful diabetic neuropathy (i.e. PD 1) caused rapid NPC neurodegeneration in distal fine processes after 15 minutes' incubation. After 20-25 minutes exposure, proximally-located processes extending from cell bodies had undergone dendritic simplification and beading. After 65 minutes, several NPC cell bodies appeared highly refractile- a characteristic of dying cells. Pre-incubation (for 5 minutes) with M100907 at $2 \mu \mathrm{M}$ concentrations completely prevented early NPC neurodegeneration (during 30 minutes' observation) in identical concentrations ( $80 \mathrm{nM})$ of the PD, Pt 1 autoantibodies. Ten-fold lower M100907 (200 nM) concentrations partially protected against PD, Pt 1 autoantibody-induced early NPC neuro degeneration in proximal dendrites. Twenty nanomolar M100907 had no significant protective effect in either proximallyor distally-located processes.

\section{Effects of diabetic autoantibodies on neuroblastoma cells morphology and survival:}

N2a

\section{Neurite retraction}

The effect of IgG autoantibodies on acute length- shortening in N2A neuroblastoma thin dendrites was examined at five -minute intervals using time-lapse microscopy (Figure 1). Significant neurite retraction was evident as early as tenfifteen minutes after application of 10 microgram per milliliter concentration of diabetic autoantibodies from two different patients (Figure 1).After 25 minutes, identical concentration of Pt 1 (a morbidly obese diabetic patient (BMI $48 \mathrm{~kg} / \mathrm{m} 2$ ) suffering with nephropathy, depression, obstructive sleep apnea, painful neuropathy, and atrial fibrillation) IgG caused mean N2A thin neurite length reduction of $68 \%$ (average of ten cells each having 2-10 processes, not shown in Figure 1). Pre-incubation (for 5

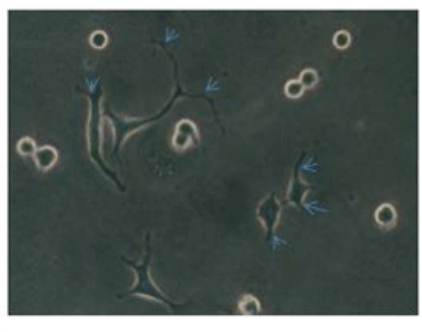

C

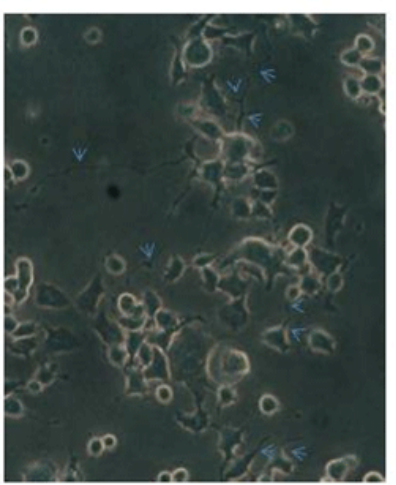

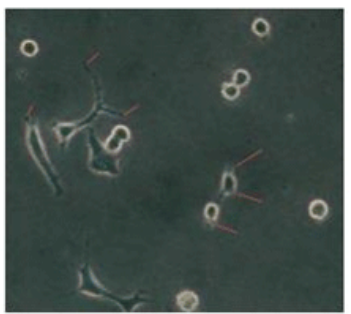

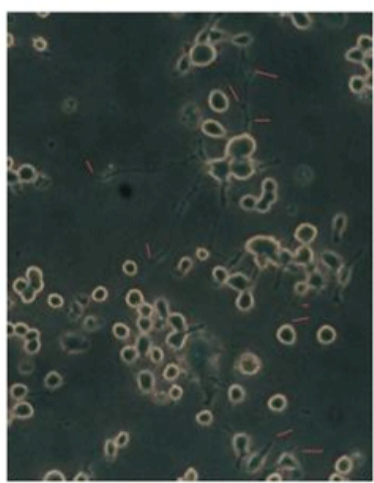

Figure 1: Inhibition of N2a neurite outgrowth by diabetic autoantibodies. Ten microgram per milliliter concentrations of diabetic autoantibodies from two different patients were incubated with N2A cells and microscopic images (200 X) magnification were acquired after 15 minutes.

(B) Or 10 minutes (D) compared to the respective baseline images (A, C) in cells before autoantibody treatment. Blue arrows $(A, C)$ and orange lines (B D) indicate corresponding points along a particular neurite segment at which acute length-shortening will have or already had occurred. Neuroblastoma cell bodies appeared contracted and displayed increased refractility (B, D) following autoantibody exposure. Loss of dendritic branches, i.e. dendritic simplification was evident in a dendrite segment after ten minutes exposure to diabetic painful neuropathy, atrial fibrillation patient autoantibodies (uppermost three blue arrows in $\mathrm{C}$ vs. uppermost three orange lines in D).

minutes) with $200 \mathrm{nM}$ concentrations of M100907, a highly selective 5-HT2A receptor antagonist, was $\sim 94 \%$ protective against IgG-induced process length reduction (assessed after 25 minutes' exposure time). M100907 at (5 -2000 nM) concentrations caused a dose-dependent inhibition of process length shortening in identical concentrations of Pt 1 IgG (Figure 2A). A log-log graph of the percent change in process length versus M100907 concentration was linear, had a slope of unity (Figure 2B) and IC50 = $39 \mathrm{nM}$. Ketanserin, a moderate affinity, selective 5-HT2A receptor antagonist, and spiperone, a high affinity, less selective 5-HT2AR antagonist, both caused dose-dependent inhibition of Pt 1,IgG-induced neurite length shortening (Figure 3A-B). The IC50 calculated from log-log plots for ketanserin and spiperone were $215 \mathrm{nM}$ and $50 \mathrm{nM}$, respectively, consistent with the order of binding affinities of the three antagonists for the 5HT2A receptor:, i.e. M100907 > spiperone > ketanserin [12].

Saturating $(1 \mu \mathrm{M})$ concentrations of 2,5-dimethoxy-4iodoamphetamine (DOI), a 5HT-2A receptor agonist, caused 


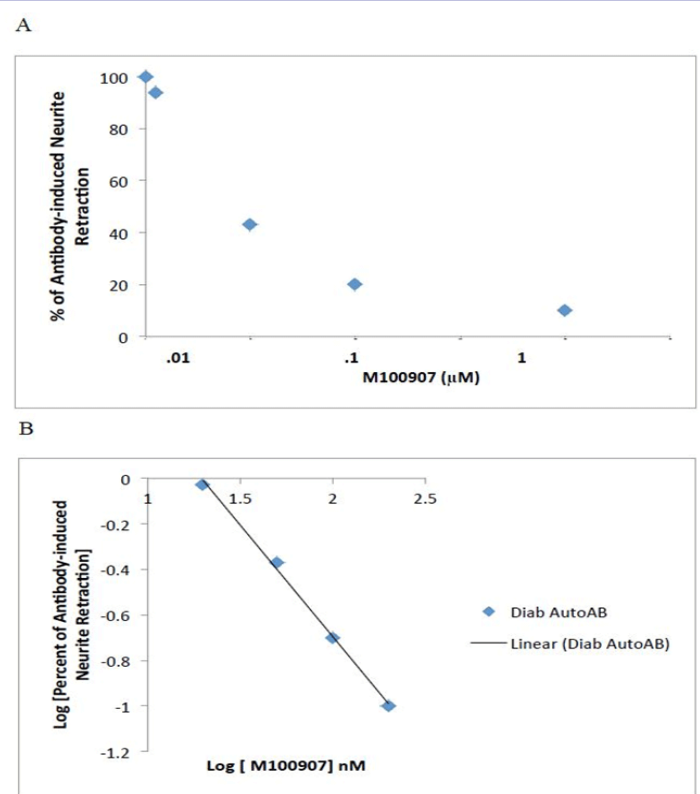

Figure 2: Dose-dependent inhibition of diabetic autoantibody, N2a neurite retraction by M100907, a highly selective 5-HT2AR antagonist. Patient 1 autoantibodies $(10 \mu \mathrm{g} / \mathrm{mL})$ were incubated with N2A cells in the presence or absence of (5-2000 nM) concentrations of M100907.

A)Percent of autoantibody-induced neurite retraction was determined as described in Methods.

B) Log-log plot of (\% of antibody retraction) vs. log M100907 concentration is linear with a slope of unity indicative of a homogeneous population of 5-HT-2A receptors.
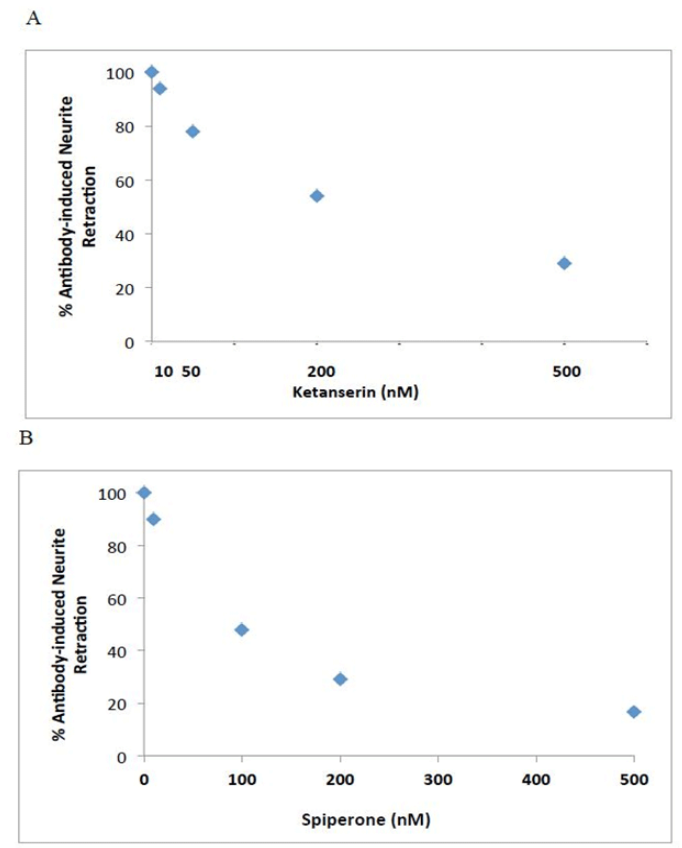

Figure 3: Dose-dependent inhibition of diabetic autoantibody (Pt 1, 10 $\mu \mathrm{g} / \mathrm{mL}$ ), N2A neurite retraction by A) ketanserin, a selective 5-HT2AR antagonist, or B) spiperone, a high affinity, less selective 5-HT2AR antagonist. Percent of autoantibody-induced neurite retraction was determined as described in Methods.
$10-15 \%$ acute length-shortening in N2a neuroblastoma cell processes after 15 minutes. After 25 minutes, process length shortening began to reverse and after 45 , and 60 minutes process length had fully reverted to baseline levels, consistent with DOIinduced desensitization [13]. Contraction in N2a cell bodies (which was evident after 15 mins) had also partially reversed after 45 minutes continuous exposure to $(1 \mu \mathrm{M})$ DOI. Taken together, these results suggest involvement of 5-HT2A receptors in the mechanism of diabetic autoantibody-induced N2a neurite retraction.

\section{N2A cell survival}

Co-incubation of N2a neuroblastoma cells with $200 \mathrm{nM}$ concentrations of the selective 5 -HT2A receptor antagonist M100907 completely protected against diabetic autoantibodiesinduced accelerated $\mathrm{N} 2 \mathrm{a}$ cell death $(\mathrm{n}=4$ different diabetic patients; $\mathrm{P}<0.001$ Fig 4A). M100907 at identical (200 nM) concentrations alone had no significant effect on $\mathrm{N} 2 \mathrm{a}$ cell survival (Figure 4A). Ketanserin (200 nM) or the selective alpha-1 adrenergic antagonist prazosin (100 or $200 \mathrm{nM})$ did not significantly protect against neuroblastoma cell death induced by identical concentrations of diabetic autoantibodies $(n=3$ patients) (Figure 4B-C). Ketanserin (200 nM) or prazosin (100 or $200 \mathrm{nM}$ ) alone did not significantly affect N2A cell survival (Figure 4B-C). Spiperone (200 nM) significantly protected (88\%) against diabetes autoantibodies-induced N2a cell death $(\mathrm{n}=2$, data not shown in Figure 4). These data suggest involvement of 5-HT2A receptors in diabetic autoantibody-induced accelerated neuroblastoma cell death. Saturating concentrations $(1 \mu \mathrm{M})$ of the 5-HT2R agonist DOI (2,5-dimethoxy-4-iodoamphetamine) alone caused dose-dependent modest decrease(s) in N2A cell survival (Figure 5B). Co-incubation of DOI $(1 \mu \mathrm{M})$ together with $10 \mathrm{nM}$ concentrations of diabetic autoantibodies significantly protected against autoantibody-induced death in a fraction of the cells (Figure 5B) consistent with DOI-induced desensitization in a fraction of cells. Dose-response curves indicated that fifty to onehundred fold lower concentrations (10-20 nM) of diabetic IgG autoantibodies caused an equivalent level of N2a cell death (after 24 hours) as saturating $(1 \mu \mathrm{M})$ concentrations of DOI (Fig $5 \mathrm{~A})$, indicative that diabetic autoantibodies caused enhanced longlasting 5-HT2AR activation via unknown mechanisms.

\section{Mechanism of diabetic autoantibody-induced N2A neurite retraction}

GPCRs belonging to the 5-HT2 family of serotonin receptors typically couple to the Galpha q/11 subfamily of heterotrimeric G-proteins [14]. Gaq/11 activation causes phospholipase C (PLC) activation resulting in increased intracellular $\mathrm{Ca} 2+$ via inositol triphosphate (IP3)-mediated release from endoplasmic reticulum stores [14]. We used specific antagonists of the PLC/ IP3/Ca2+ pathway to test for possible involvement of a Gaq/11 -coupled mechanism underlying autoantibody-induced neurite retraction. Autoantibody-induced neurite retraction was nearly completed (85-90\%) blocked by pre-incubation (15 minutes) with $(1 \mu \mathrm{M})$ concentrations of U73211, a specific phospholipase C-gamma (PLC- $\gamma$ ) inhibitor (Table 4). It was completely 
blocked by pre-incubations with $(50 \mu \mathrm{M})$ concentrations of the inositol 1,4,5-trisphosphate (IP3) receptor antagonist 2-aminoethoxydiphenyl borate (2-APB) or $(10 \mu \mathrm{M})$ RhoA/ Rho kinase inhibitor Y27632 (Table 4). It was also completely blocked by pre-incubation (15 mins) with $(30 \mu \mathrm{M}) \mathrm{Ca} 2+$ chelator, BAPTA-AM. Ten micromolar concentrations of Y27632 and fifty micromolar 2-APB not only completely prevented autoantibody neurite retraction, but they caused significant neurite lengthening consistent with suppression of basal GPCR, Gaq/11 activation . Taken together, these data suggest involvement of the PLC- $\gamma /$ IP3R/Ca2+ pathway (which positively couples to Gaq/11 and 5-HT-2A receptor) in the mechanism of diabetes autoantibodiesdependent neurite retraction.
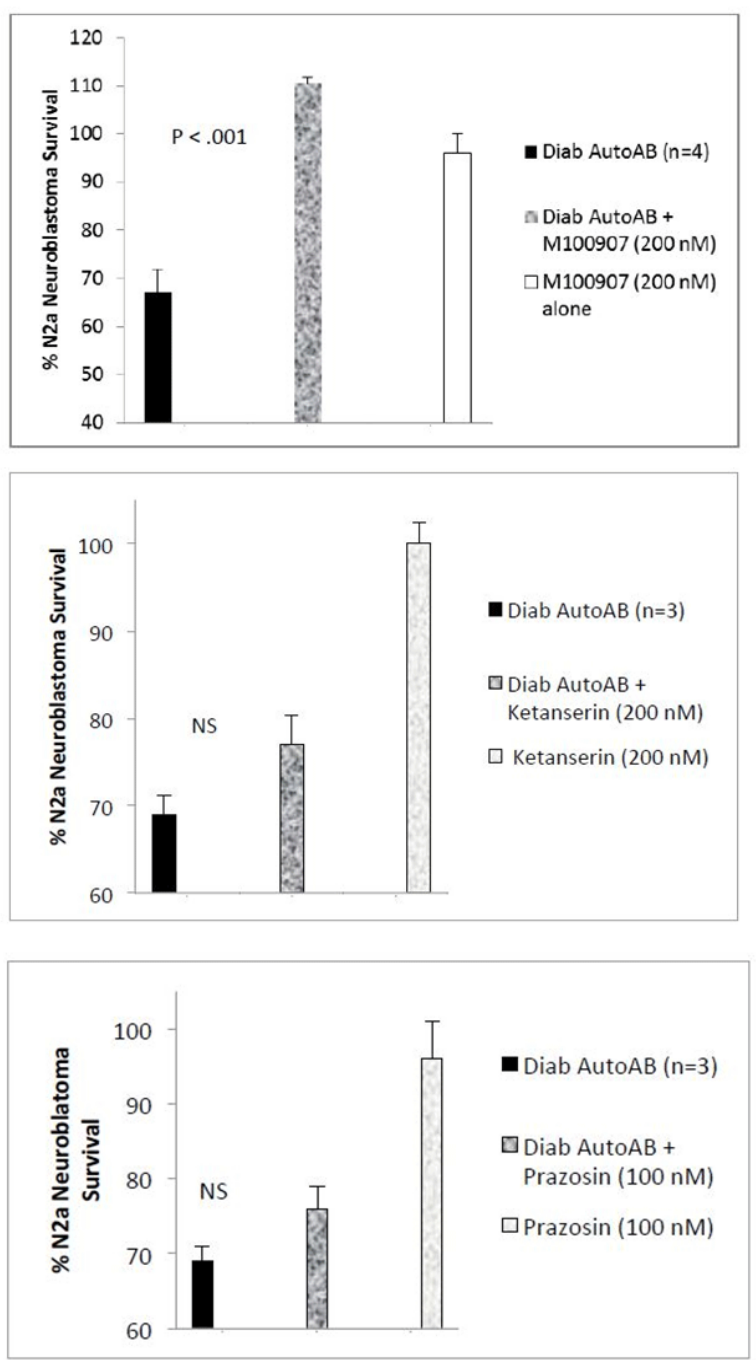

Figure 4: Effect of GPCR antagonists on diabetic autoantibody-induced accelerated N2A cell loss. N2A neuroblastoma cell autoantibody- induced accelerated loss was A) completely prevented by co-incubation with $200 \mathrm{nM}$ concentrations of M100907, B-C) lack of significant protection by co-incubation with $200 \mathrm{nM}$ ketanserin(B) or $100 \mathrm{nM}$ prazo$\sin (\mathrm{C})$. N2A cells were incubated in the presence of absence of specific GPCR antagonists for 24 hours at 37 degrees C; cell number was determined as described in Methods. Results are the mean \pm SEM. NS- not statistically significant.
N2A accelerated cell death (after 8 hours incubation) with autoantibodies from three different diabetic patients was significantly prevented by co-incubation with (non-toxic) $1 \mu \mathrm{M}$ concentrations of the IP3R inhibitor 2-APB (Figure 5C) suggesting involvement of sustained $\mathrm{Ca} 2+$ release in the mechanism of neuroblastoma cell death.
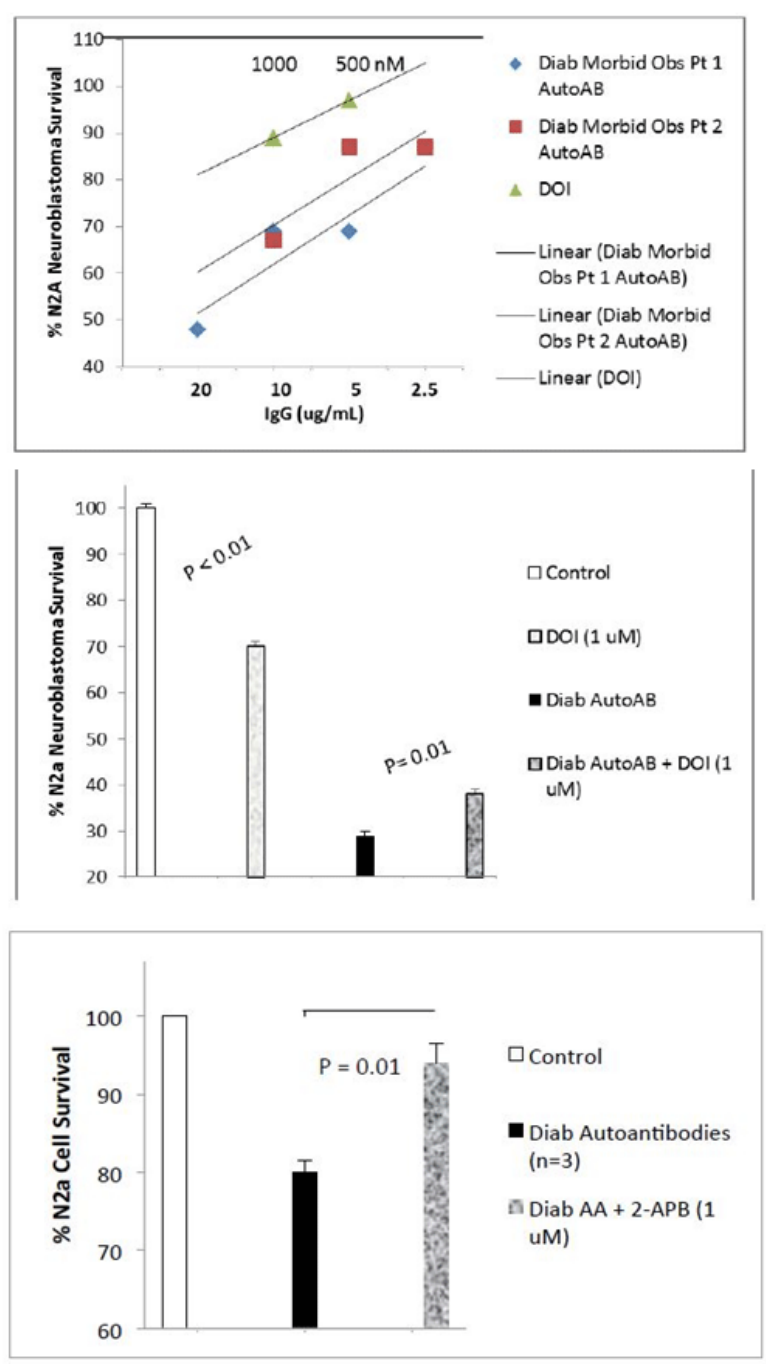

Figure 5: Effect of 5-HT-2AR agonist (DOI) on N2A survival in the absence (A-B) or (B) presence of diabetic autoantibodies; C) effect of 2-APB (inositol triphosphate 3 receptor antagonist) on N2A survival in the presence of diabetic autoantibodies. N2A cells were incubated for 24 hours (A-B) or for 8 hours (C) at 37 degrees C; cell number was determined as described in Methods. Results are the mean \pm SEM.

\section{Effect of diabetic autoantibodies on endothelial cell survival}

M100907 at (200-2000 nM) concentrations completely protected endothelial cells from cell death induced by diabetic Parkinson's disease $(10 \mu \mathrm{g} / \mathrm{mL})$ (Figure 6A) or diabetic depression autoantibodies (not shown in Figure 6A). Lower M100907 concentrations (50-100 nM) caused dose-dependent significant (16-27\%) protection (Figure 6B) against diabetic 
autoantibodies - induced EC death ( $\mathrm{n}=$ three different patients). The autoantibodies $(10 \mu \mathrm{g} / \mathrm{mL})$ in a Parkinson's disease-sufferer (PD2), who also had autoimmune thyroid disease and diabetic nephropathy promoted endothelial cell proliferation (Figure 6C); and the modest EC proliferation was completely neutralized by $200 \mathrm{nM}$ concentrations of M100907 (Figure 6C). After brief storage (7 days at 4 degrees C), re-assay of an identical concentration of the PD2 autoantibodies revealed EC inhibitory activity which was sensitive to neutralization (22\%) by low ( $25 \mathrm{nM}$ ) concentrations of M100907 (Figure 7A). Substantially higher concentrations of ketanserin $(100 \mathrm{nM})$ or prazosin $(1 \mu \mathrm{M})$ alone had no significant effect on the EC inhibitory activity in PD2 autoantibodies (Figure
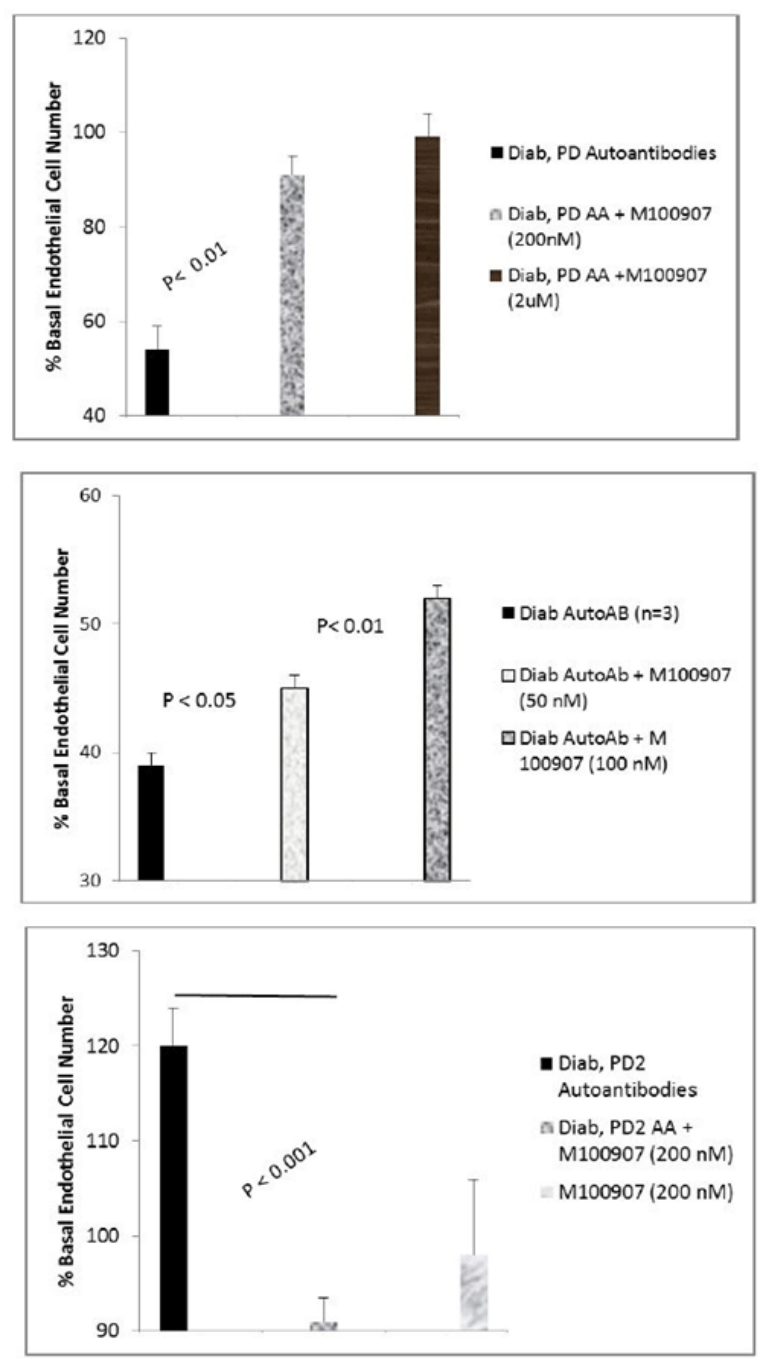

Figure 6: Effect of highly selective 5-HT-2AR antagonist M100907 on endothelial cell survival induced by diabetic autoantibodies. M100907 dose-dependently protected against $(A, B)$ endothelial cell survival inhibitory effect in Parkinson's disease, pt 1 autoantibodies $(10 \mu \mathrm{g} / \mathrm{mL})$. C) M100907 (200 nM) completely neutralized EC stimulation from Parkinson's disease, pt 2 autoantibodies $(10 \mu \mathrm{g} / \mathrm{mL})$. Bovine pulmonary artery endothelial cells were incubated in the presence or absence of Parkinson's disease autoantibodies for 48 hours at 37 degrees C; cell number was determined as described in Methods. Results are the mean \pm SEM.
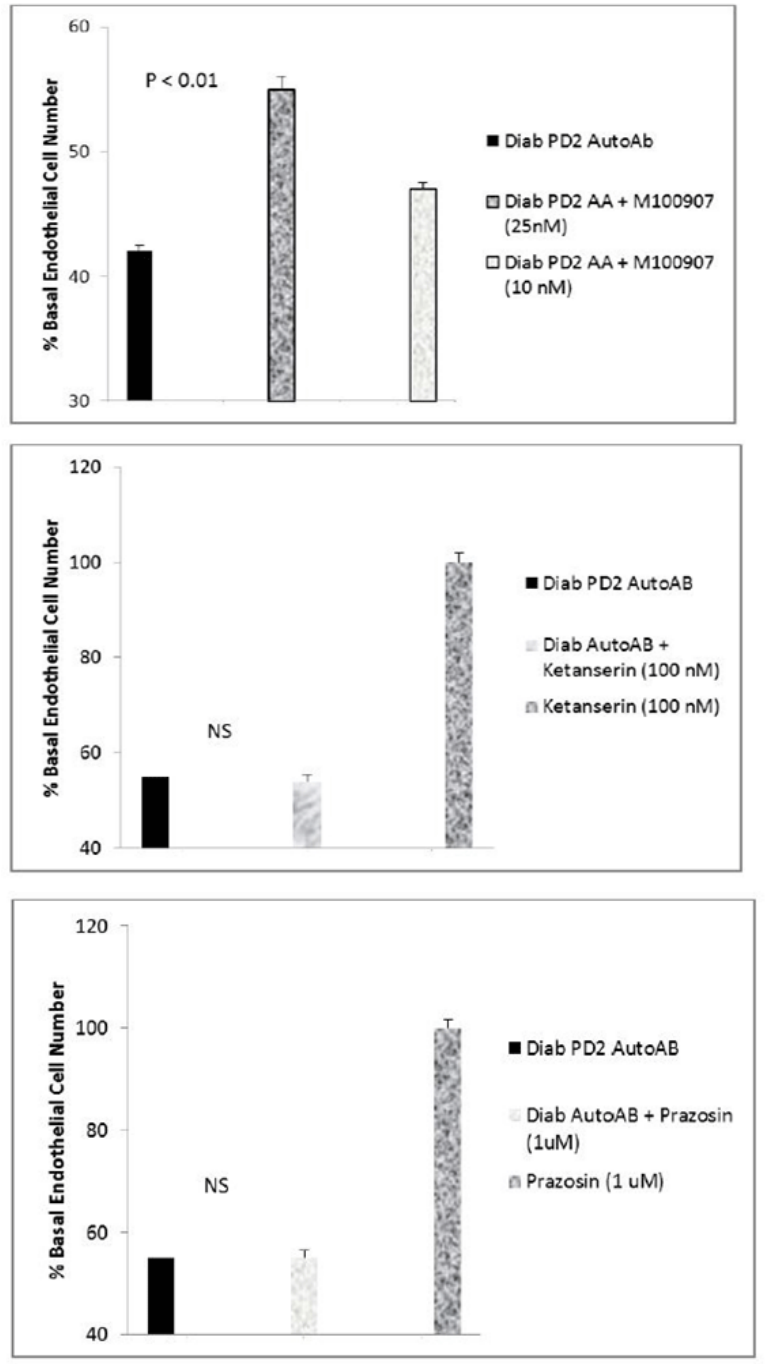

Figure 7: Effect of M100907 (A) or other GPCR antagonists (B,C) on Parkinson's disease Pt 2 autoantibody-induced inhibition of endothelial cell survival. Bovine pulmonary artery endothelial cells were incubated in the presence or absence of Parkinson's disease, Pt 2 autoantibodies $(10 \mu \mathrm{g} / \mathrm{mL})$ for 48 hours at 37 degrees C; cell number was determined as described in Methods. Results are the mean \pm SEM. NS- not statistically significant.

$7 \mathrm{~B}, \mathrm{C})$. These data suggest involvement of a 5HT-2 receptor in the inhibitory effects of diabetic neurovascular autoantibodies on EC survival.

Vascular cells express endothelin A receptor (ET-AR), angiotensin II, type 1 receptor (AT-1R) and metabotropic glutamate 5 receptor (mGlu-5R). Saturating concentrations (10 $\mu \mathrm{M})$ of each of the specific GPCR antagonists: bosentan (ET-AR), losartan (AT-1R) or CTEP (mGlu-5R) had a modest inhibitory effect on autoantibody-mediated EC survival (Table 3) consistent with GPCR cross-talk, but lack of direct involvement of these receptors in autoantibody-mediated EC survival inhibition. 

Ca2+ Pathway

\section{Discussion}

The present evidence is the first to suggest that autoantibodies in subsets of diabetes suffering with nephropathy, atrial fibrillation, morbid obesity, depression, and/or Parkinson's disease mediate neural- and endothelial cell- inhibitory effects via 5-hydroxytryptamine- 2 receptors. The 5-hydroxytryptamine receptor-2A (5-HT2AR) is expressed in neuroblastoma cells [15]; and 5HT-2AR is the predominant subtype which is highly concentrated in brain regions involved in perception, mood, learning and memory, pain, cognition, appetite, and sleep/ wakefulness [16]. The 5HT-2A receptor is a target of atypical antipsychotic drugs [17], and anti-depressant medications, such as amitriptyline [18], the latter is also useful in the treatment of painful diabetic neuropathy.

We previously reported highly potent neural- and ECinhibitory autoantibodies enriched in subsets of diabetes having co-morbid depression, schizophrenia and/or painful neuropathy compared to age-matched control diabetic populations lacking these specific complications (Table 1). The present finding that three different 5HT-2AR antagonists prevented autoantibodyinduced acute N2A neurite retraction and displayed an order of potency (M100907> spiperone> ketanserin) which mirrored their relative affinities for the 5-HT-2AR [12] suggests involvement of 5-HT-2A receptors in diabetic autoantibody-induced neural inhibitory effects.

The 5-HT-2A receptor couples to a $\mathrm{G} \alpha \mathrm{q} / 11$ subclass of heterotrimeric $G$ proteins in neurons and other cell types [14].

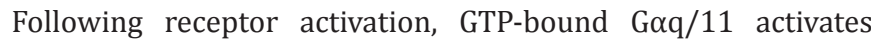
phospholipase C (PLC- $\gamma$ ) which in turn hydrolyzes PIP2 to yield inositol triphosphate (IP3) and diacylglycerol (DAG). IP3 causes release of $\mathrm{Ca} 2+$ from intracellular stores, the latter plays a role in the activation of protein kinase $\mathrm{C}$. In the current study, specific inhibitors of phospholipase C (PLC- $\gamma$ ), IP3R and an intracellular calcium chelator each nearly or completely prevented acute autoantibody-induced neurite retraction. The selective RhoA/ ROCK inhibitor Y27632 $(10 \mu \mathrm{M})$ also completed prevented acute neurite retraction consistent with results in previous studies $[5,6,7,8,9]$. The precise mechanism linking PLC/IP3R/ $\mathrm{Ca} 2+$ activation with RhoA/ROCK-signaling in N2A cells is not clear, however, evidence from other laboratories indicates that ROCK activation can occur through the coordinated action(s) of activated $\mathrm{G} \alpha \mathrm{q} / 11$ and beta $(\beta)$ - arrestin-1 [19].

Large increases in intracellular $\mathrm{Ca} 2+$ are known to be toxic to neurons [20]. Substantial protection against accelerated N2A cell death by nontoxic concentration of 2-APB $(1 \mu \mathrm{M})$ suggests an important role for release of $\mathrm{Ca} 2+$ from sequestered intracellular stores in the mechanism of accelerated neuroblastoma cell loss.

A striking feature of autoantibody-induced neurite retraction was the absence of its spontaneous cessation, e.g. as occurred in N2A cells exposed to high concentrations of the hallucinogenic 5-HT2A agonist 2,5-dimethoxy-4-iodoamphetamine, (DOI). Beta arrestin -1 and -2 knock-out-bearing mouse embryonic fibroblasts displayed 'enhanced agonism' at both the angiotensin II receptor and beta adrenergic receptors [21] consistent with a general role for $\beta$-arrestins in GPCR- desensitization [14]. The mechanism of diabetic autoantibodies 'long-lasting agonism' at the 5-HT-2AR is unknown. One possibility (which requires more study) is that it may interfere with $\beta$-arrestin binding to regulatory domains located in the third intracellular loop of GPCR [22]. Dimeric or oligomeric forms of IgG autoantibodies might preferentially target 5-HT-2A receptor heterodimers in the membrane which (in some cases) are comprised by highly stable non-covalent interactions between oppositely-charged amino acid residues in the intracellular domains of physically neighboring GPCRs [23]. Stable heteromers involving GPCRs positively coupled to Gq/11 heterotrimer $\mathrm{G}$ proteins may contribute to sustained activation of PLC/IP3R/Ca2+ pathway causing long-lasting Ca2+ release (>10 minutes) similar to that which was observed in different subsets of diabetic autoantibodies [8].

The 5-hydroxytryptamine-2A receptor can activate multiple, different signaling pathways depending (in part) on differences in the chemical structure(s) among particular agonist ligands [14]. The ability of a given ligand to activate a specific intracellular signaling pathway downstream of 5-HT-2AR binding has been referred to as 'biased agonism' or 'functional selectivity [14].' In a prior report in three hundred and five diabetic nephropathy patients from the VA NEPHRON D randomized clinical trial, patients harboring less- inhibitory or mildly-stimulatory EC plasma autoantibodies were at substantially higher risk of experiencing a composite chronic kidney disease (CKD) endpoint, (i.e. significant decline in renal function, end-stage renal disease or death), compared to patients harboring strongly- inhibitory EC plasma autoantibodies [3]. Here, the protein-A eluate in a diabetic CKD, Parkinson's disease patient (i.e. PD2) contained components which evoked either mild EC stimulation or potent EC inhibition and unexpectedly, both kinds of responses were blocked by M100907 suggesting 5-HT-2AR-mediated functional selectivity. Although the precise make-up of the autoantibody ligand(s) is unknown, evidence from prior studies suggests that EC stimulation may have resulted from an immune complex ligand. Autoantibodies in four additional diabetic nephropathy/ CKD patients evoked initial EC stimulation and upon re-assay following brief storage, EC inhibition (M. Zimering, unpublished data). These data suggest that immune complexes in diabetic CKD protein-A eluates were less stable (in dilute solution) compared to component IgG. More study is needed to determine whether diabetic CKD autoantibodies may also induce proliferation in mesangial cells, via 5-HT-2A receptors coupled to ERK signaling [24].

The pharmacologic profile of antagonism of diabetic autoantibody-induced accelerated endothelial cell death suggests possible involvement of additional receptor subtypes besides 5-HT-2A. For example, $100 \mathrm{nM}$ ketanserin did not protect against autoantibody-induced endothelial cell death despite having low nanomolar affinity at the 5HT-2A receptor. Since 5-HT-2BR is the predominant 5HT receptor subtype expressed on pulmonary artery endothelial cells [26] and ketanserin has low micro-molar affinity at the 5-HT-2B receptor [25], these data suggest possible involvement of the 5-HT-2B receptor subtype in autoantibody- 
mediated accelerated endothelial cell death.

Adult neurogenesis in the dentate gyrus has been shown to correlate with the efficacy of clinical response(s) to various anti-depressant treatments [27]. The present data showing that diabetic autoantibodies inhibitory effects on DG NPC cells could be completely prevented by the selective 5-HT-2AR antagonist M100907 is of interest. Adult DG neural progenitor cells express multiple different 5-HT-1 and 5-HT-2 receptor subtypes [28]. The inhibitory effects of long-lasting 5-HT-2R agonist diabetic depression autoantibodies are consistent with a report that chronic (7 days) administration of a 5-HT-2R agonist, $\alpha$-methyl5-HT, suppressed newborn DG neuron survival in adult female C57Bl/6 mice [28].

The ontogeny of agonist 5-hydroxytryptamine-2A receptor autoantibodies is unknown. One possibility is endothelial injury which releases sequestered auto-antigens, e.g. basic fibroblast growth factor (bFGF) [29], whose increased concentration in plasma correlated with the spontaneous appearance of agonist FGF-like autoantibodies in various pathologies including cancer subsets [11, 30] and obese type 2 diabetes [31]. Vascular injury also causes platelets to release serotonin together with polyphosphates, the latter of which, like heparin, is a highly anionic substance capable of binding and protecting (locallyreleased) bFGF from inactivation [32]. Since platelet activation increases in obesity and inflammation [33], it is possible that repeated vascular injury through the release of sequestered antigens promotes immune response(s) (in susceptible individuals) leading to the formation of agonist 5-HT-2A receptor autoantibodies. Kidney was reported to have among the highest 5-HT-2R mRNA expression levels among peripheral organs [25]. Thus it is possible that 5-HT-2R released through ongoing renal endothelial damage (e.g. in diabetic nephropathy populations) may contribute (in part) to the development of agonist 5-HT-2 receptor autoantibodies.

In summary, diabetic autoantibodies from patients suffering with nephropathy, and/or depression, atrial fibrillation, painful neuropathy or another neurodegenerative disease caused N2A neurite retraction and accelerated neuron loss through a mechanism involving 5-HT-2A receptors positively coupled to the PLC/IP3/Ca2+ pathway. These findings have relevance for understanding possible role(s) for agonist 5-hydroxytryptamine$2 \mathrm{~A}$ receptor autoantibodies in pathophysiology in diabetesrelated complications affecting brain regions and peripheral organs in which the 5-HT-2 receptor is highly concentrated.

\section{Acknowledgements}

Dr. Janet Alder, Dr. Smita Thakker-Varia, and Shavonne Teng (Department of Neuroscience \& Cell Biology, Rutgers-Robert Wood Johnson Medical School, Piscataway, New Jersey) for providing the mouse $\mathrm{N} 2 \mathrm{~A}$ neuroblastoma and rat dentate gyrus neural progenitor cells used in these experiments. Veterans Biomedical Research Institute (East Orange, New Jersey) for grant support to MBZ.

\section{References}

1. Deckert T, Feldt-Rasmussen B, Borch-Johnsen K, Jensen T, KofoedEnevoldsen A. Albuminuria reflects widespread vascular damage. The Steno hypothesis. Diabetologia 1989;32(4):219-226.

2. Stehouwer CD, Gall MA, Twisk JW, Knudsen E, Emeis JJ, Parving HH. Increased urinary albumin excretion, endothelial dysfunction, and chronic low-grade inflammation in type 2 diabetes: progressive, interrelated, and independently associated with risk of death. Diabetes. 2002;51(4):1157-1165. Doi: 10.2337/diabetes. 51.4.1157

3. Zimering MB, Zhang, JH, Guarino P, Emanuele N, Mc Cullogh P, Fried L et al. Endothelial cell autoantibodies in predicting declining renal function, end-stage renal disease or death in adult type 2 diabetic nephropathy Front. Endocrinol. 2014;5:128. Doi: 10.3389/ fendo.2014.00128

4. Fried LF, Emanuele N, Zhang JH, Brophy M, Conner TA, Duckworth W, et al. Combined angiotensin inhibition for the treatment of diabetic nephropathy. N Engl J Med. 2013;369(20):1892-1903. Doi: 10.1056/ NEJMoa1303154

5. Zimering MB, Behnke JA, Thakker-Varia S, Alder J. Autoantibodies in human diabetic depression inhibit adult neural progenitor cells in vitro and induce depressive-like behavior in rodents. J Endocrinol Diab 2015;2(2):1-11.

6. Zimering MB, Mirkovic N, Pandya M, Zimering JH, Behnke JA, et al. Toxic Immunoglobulin Light Chain Autoantibodies are Associated with a Cluster of Severe Complications in Older Adult Type 2 Diabetes. J Endocrinol Diab 2016:3(1):1-14.

7. Zimering MB, Alder J, Pan Z, and Donnelly RJ. Anti-endothelial and anti-neuronal effects from auto-antibodies in subsets of adult diabetes having a cluster of micro vascular complications. Diabetes Res. Clin Pract. 2011:93(1);95-105.

8. Zimering $\mathrm{MB}$, Pan Z. Increased Neuronal Depolarization Evoked by Autoantibodies in Diabetic Obstructive Sleep Apnea: Role for Inflammatory Protease(S) in Generation of Neurotoxic Immunoglobulin Fragment. J Endocrinol Diab. 2016:4(1):1-10.

9. Zimering MB, Moritz TE and Donnelly RJ. Anti-neurotrophic effects from autoantibodies in adult diabetes having primary open angle glaucoma or dementia. Front. Endocrinol. 2013:4;58. Doi: 10.3389/ fendo.2013.00058

10.Zimering MB, and Pan Z. Autoantibodies in type 2 diabetes induce stress fiber formation and apoptosis in endothelial cells. J Clin Endocrinol Metab. 2009:94(6);2171-2177.

11.Zimering MB, Thakker-Varia S. Increased fibroblast growth factor -like autoantibodies in serum from a subset of patients with cancerassociated hypercalcemia. Life Sci. 2002;71(25);2939-2959.

12. Roth BL, Driscol J. “PDSP Ki Database”. Psychoactive Drug Screening Program (PDSP). University of North Carolina at Chapel Hill and the United States National Institute of Mental Health. 2012.

13. Berry SA, Shah MC, Khan N, Roth BL. Rapid agonist-induced internalization of the 5 -hydroxytryptamine2A receptor occurs via the endosome pathway in vitro. Mol Pharmacol. 1996;50(2):306-313. 


\section{Ca2+ Pathway}

14. Bohn LM, Schmid CL. Serotonin receptor signaling and regulation $\beta$ via -arrestins. Crit Rev Biochem Mol Biol. 2010;45(6):555-566.

15. Marinova Z, Walitza S, Grunblatt E. 5-HT2A serotonin receptor agonist DOI alleviates cytotoxicity in neuroblastoma cells: role of the ERK pathway. Prog Neuropsychopharmacol Biol Psychiatry. 2013:44;6472 .

16.Xu T, Pandey SC. Cellular localization of serotonin (2A) (5HT (2A)) receptors in the rat brain. Brain Res Bull. 2000;51(6):499-505.

17. Kehne JH, Baron BM, Carr AA, et al. Preclinical characterization of the potential of the putative atypical antipsychotic MDL 100,907 as a potent 5-HT2A antagonist with a favorable CNS safety profile. Journal of Pharmacology and Experimental Therapeutics. 1996:277(2);968981.

18. Hisaoka K1, Tsuchioka M, Yano R, Maeda N, Kajitani N, Morioka $\mathrm{N}$, et al. Tricyclic antidepressant amitriptyline activates fibroblast growth factor receptor signaling in glial cells: involvement in glial cell line-derived neurotrophic factor production. J Biol Chem. 2011;286(24):21118-21128

19. Barnes WG, Reiter E, Violin JD, Ren XR, Milligan G, Lefkowitz RJ. Beta-Arrestin 1 and Galphaq/11 coordinately activate RhoA and stress fiber formation following receptor stimulation. J Biol Chem. 2005;280(9):8041-50. Doi: 10.1074/jbc.M412924200

20. Mattson MP, Guthrie PB, Kater SB. Intrinsic factors in the selective vulnerability of hippocampal pyramidal neurons. Prog Clin Biol Res. 1989:317; 333-351.

21. Kohout TA, Lin FS, Perry SJ, Conner DA, Lefkowitz RJ. Beta-Arrestin 1 and 2 differentially regulate heptahelical receptor signaling and trafficking. Proc Natl Acad Sci U S A. 2001;98(4):1601-1616

22. Gelber EI, Kroeze WK, Willins DL, Gray JA, Sinar CA, Hyde EG, et al. Structure and function of the third intracellular loop of the 5-hydroxytryptamine2A receptor: the third intracellular loop is alphahelical and binds purified arrestins. J Neurochem. 1999;72(5):22062214

23. Moutkine I, Quentin E, Guiard B, Maroteaux L, Doly S. Heterodimers of serotonin receptor subtypes 2 are driven by 5 -HT2C protomers. J Biol Chem. 2017;292(15):6352-6368.
24.Grewar JS, Mukhin YV, Garnovskaya MN, Raymond JR, Greene EL. Serotonin 5-HT2A receptor induced TGF-beta1 expression in mesangial cells via ERK: proliferative and fibrotic signals. Am J Physiol. 1999;276(6 pt2):F922-930.

25. Bonhaus DW, Bach C, DeSouza A, Salazar FH, Matsuoka BD, Zuppan $\mathrm{P}$, et al. The pharmacology and distribution of human 5-hydroxytryptamine2B (5-HT2B) receptor gene products: comparison with 5-HT2A and 5-HT2C receptors. Br J Pharmacol. 1995;115(4):622-628.

26. Ullmer C, Boddeke HG, Schmuck K, Lübbert H. 5-HT2B receptormediated calcium release from ryanodine-sensitive intracellular stores in human pulmonary artery endothelial cells. Br J Pharmacol. 1996;117(6):1081-1088.

27.Warner-Schmidt JL, Duman RS. Hippocampal neurogenesis: opposing effects of stress and antidepressant treatment. Hippocampus. 2006;16(3):239-429.

28. Klempin F, Babu H, De Pietri Tonelli D, Alarcon E, Fabel K and Kempermann G. Oppositional effects of serotonin receptors 5-HT1a, 2, and $2 \mathrm{c}$ in the regulation of adult hippocampal neurogenesis. Front Mol Neurosci. 2010:3:14. Doi: 10.3389 /fnmol.2010.00014

29. Gajdusek CM, Carbon S. Injury-induced release of basic fibroblast growth factor from bovine aortic endothelium. J Cell Physiol 1989;139(3):570-579.

30.Zimering MB, Alder J, Thakker-Varia S. Neurotrophic effects of fibroblast growth factor-like autoantibodies in serum from three patients with breast cancer. Brain Research. 2009:1251;276-286. Doi: 10.1016/j.brainres.2008.11.035

31.Zimering MB, Anderson RJ, Ge L, Moritz TE, Duckworth WC, Investigators for the VADT. Basic fibroblast growth factor predicts cardiovascular disease occurrence in participants from the veterans affairs diabetes trial. Front Endocrinol (Lausanne) 2013:4;183.

32. Shiba T, Nishimura D, Kawazoe Y, Onodera Y, Tsutsumi K, Nakamura R, et al. Modulation of mitogenic activity of fibroblast growth factors by inorganic polyphosphate. J Biol Chem. 2003;278:26788-26792.

33. Lurie A. Inflammation, oxidative stress, and procoagulant and thrombotic activity in adults with obstructive sleep apnea. Adv Cardiol. 2011;46:43-66. Doi: 10.1159/000325105 